\title{
Effect of Deliberate Ingestion of Organophosphate Pesticide on Distortion Product Oto-Acoustic Emissions (DPOAE)
}

\author{
Srihimaja Nandamudi ${ }^{1}$, Vinaya Ashok Murthy ${ }^{2}$, Yugandhar Ramakrishna ${ }^{2}$, Tejaswini Boreddy ${ }^{3}$, \\ Savalam Bujjetha Rathna Kumar ${ }^{1}$, Savalam Gnana Rathna Prakash ${ }^{1}$ \\ ${ }^{1}$ Ali Yavar Jung National Institute for the Hearing Handicapped, Southern Regional Centre, Ministry of Social Justice and Empow- \\ erment, Government of India, Manovikasnagar, Secunderabad; ${ }^{2}$ People’s Education Society Institute of Medical Sciences and Re- \\ search Centre, Kuppam, Chittoor, India; ${ }^{3}$ Institute of Health Sciences (IHS), Bhubaneshwar, India. \\ Email: srihimaja.nandamudi@gmail.com, sarathna@yahoo.co.in, prakash_nihh@rediffmail.com,yuvaaslp.r@hotmail.com, \\ ashokmurthy_army@yahoo.co.in, tejuaslp@gmail.com
}

Received August $16^{\text {th }}, 2012$; revised September $18^{\text {th }}$, 2012; accepted October $4^{\text {th }}, 2012$

\begin{abstract}
Background: Oto-Acoustic Emissions (OAE) are low intensity sounds produced by the cochlea in response to an acoustic stimulus. Deliberate self-poisoning has become an increasingly common response to emotional distress in young adults (NHS, 1998). Organophosphate (OP) compounds are most commonly involved in 76\% of pesticide poisoning especially in the developing countries like India. Several pesticides are neurotoxin which could potentially affect hearing and, animal studies showed that OP treatment leads to change in hearing threshold, outer and inner hair cell loss but human studies on the same are very few. Aim: The present study was aimed at exploring the cochlear changes after self-poisoning with OP pesticides by measuring the Distortion Product Oto-Acoustic Emissions (DPOAE). Method: 114 subjects were participated in the study, with an age range of 18 - 30 years having pure tone audiometric thresholds within the normal limits and no history of middle ear pathology. They were further divided into two groups, I and II which includes self-poisoned and healthy subjects respectively. Results: The "pass" and "fail" criteria of DPOAE measures in group I were $30 \%$ and $70 \%$ respectively whereas in group II, it was found $91 \%$ and $9 \%$ respectively. Discussion: There was a significant failure in DPOAE in Group I (subjects who were self-poisoned) compared to group II (healthy subjects). The two important pathologies behind OP toxicity are the generation of Reactive Oxygen Species and the depletion of NADPH which is necessary for normal function. Conclusion: The results of the present study highlight that; the minute cochlear changes caused due to pesticide poisoning can be effectively measured through DPOAE. Further it can suggest in other clinical targets like Oto-toxicity, Noise-induced hearing loss, Meniere's disease etc., which has an effect on cochlear hair cells can be monitored through DPOAE.
\end{abstract}

Keywords: Oto-Acoustic Emissions (OAE); Organophosphate (OP); Ototoxity; Cochlear Changes

\section{Introduction}

\subsection{OP Pesticides and Suicides}

Each year, nearly 900,000 deaths are due to suicide, which accounts for more deaths than homicides and wars combined. This number is believed to be largely underestimated as suicide as a cause of death is underreported in which pesticide ingestion is one of the leading suicide methods, estimated around three million cases, resulting in an excess of 250,000 deaths [1]. Deliberate self-poisoning has become an increasingly common response to emotional distress in young adults [2]. The substances most commonly used for self-poisoning are agricultural pesticides [3,4]. Organophosphate (OP) compounds are most commonly used among them and are involved in
$76 \%$ of pesticide poisoning [5] especially in the developing countries like India and could potentially affect hearing due to their neuro-toxic nature. The principle pharmacological action of all OP compounds is the inhibition of acetyl cholinesterase, resulting in over-stimulation of acetylcholine receptors. A few case studies have pointed to acute poisoning with organophosphate (OP) insecticides as one potential cause of permanent, bilateral hearing loss [6,7]. Most of the studies in animals have demonstrated that these agents have an oto-toxic effect $[8,9]$, and in humans both peripheral hearing loss [10] and central site for the damage are induced by solvents [11].

\subsection{Otoacoustic Emissions}

Oto-Acoustic Emissions (OAE) are low intensity sounds 
produced by the cochlea in response to an acoustic stimulus [12]. A moderate intensity click stimulus or an appropriate combination of two tones can evoke outer hair cell (OHC) motility. This motility affects basilar membrane bio-mechanics, resulting in a form of intracochlear energy amplification, as well as cochlear tuning for more frequency resolution [13]. The OHC are the most vulnerable in peripheral auditory system [14] and damage to these cells leads to a reduction or absence of evoked OAE. Among patients with mild cochlear dysfunction, OAE may be recorded but amplitudes are below normal limits/absent for some or all stimulus frequencies. However, some patients (30\% of population) with abnormal OAE, consistent with cochlear dysfunction, will have normal pure-tone audiogram $[15,16]$. There is a consensus in the literature that OAE reception is highly stable $[17,18]$ and the primary targets include noise induced hearing loss [19], Meniere's disease [20, 21], oto-toxicity [22-25] etc., OAE have been suggested as a tool for the early detection of auditory damage due to diabetes [26] and noise [27]. However, there is no strong evidence that solvents induce damage to OHCs in humans.

\section{Need of the Study}

The non-invasive nature, with their ease, specificity, sensitivity, accuracy and objectivity of OAE helps in assessing the minute/sub-clinical changes in outer hair cell function. Auditory damage induced by exposure to solvents in the occupational settings has been investigated and the case reports suggested solvent-induced oto-toxicity [28]. As the literature suggests auditory damage due to solvent exposure, it leads to more serious condition due to the direct oral ingestion of OP compounds. Hence the present study needs to ascertain the function of outer hair cells in the subjects who deliberately ingested the OP pesticides. In numerous studies, researchers have reported that OAE level changes preceded behavioural hearing changes in patients receiving oto-toxic drugs, whether pure-tone threshold were tested within the conventional frequency range [29,30]. The increased susceptibility of OAEs to ototoxic damage compared to behavioural testing may reflect DPOAE sensitivity to preclinical changes in the cochlear outer hair cell function [31]. Such evidence supports the potential use of OAE testing for the early detection of solvent-induced hearing loss. It implies that when DPOAE begin to decrease before hearing threshold increases, it is possible that the test may allow us to predict future hearing loss [32]. Hence there is a need to identify the effect of deliberate ingestion of OP pesticide on DPOAE which helps to explore the cochlear changes among the individuals who manifest audiometric thresholds within normal limits.

\section{Method}

\subsection{Subjects}

A total of 114 subjects (68 males and 46 females) were taken in the study, with an age range of 18 - 30 years. All the subjects must have pure tone audiometric thresholds within the normal limits, "A" type of tympanogram and presence of acoustic reflexes; and no previous history of middle ear pathologies. These subjects were equally divided into two groups (Table 1), group-I and group-II. Group I: subjects who were self-poisoned and admitted in People's Education Society, Institute of medical sciences and research Centre, Kuppam, with in the period of a year (May, 2010-2011) were included in this group and Group II: included 57 healthy subjects.

\subsection{Instrumentation}

MAICO MA 53 audiometer with TDH 39 head phones were used to measure pure tone audiometric thresholds and MADSEN ZODIAC instrument was used for Impedance audiometric measurements and DPOAE were measured using IHS software with a single channel smart USB lite hardware. DP-gram stimulus protocol include a frequency range from about 0.5 to $8 \mathrm{KHz}$ with respect to geometric mean frequency, or 0.55 to $8.8 \mathrm{KHz}$ re f2, along with a f2/f1 ratio of 1.22, a level difference of 10 $\mathrm{dB}$, and absolute levels of $\mathrm{L} 1=65 \mathrm{dBSPL}$ and $\mathrm{L} 2=$ 55dBSPL.

\subsection{Procedure}

The subjects who were participated in the study had been undergone for otoscopic examination, Pure-tone audiometry, impedance audiometry. Group-II which included the healthy subjects was randomly selected for the participation whereas Group-I which included the subjects who had deliberate ingestion of OP pesticides for suicidal attempt were purposively selected for the study. The test procedure for the latter group was administered after

Table 1. Demographic data of the participants.

\begin{tabular}{ccc}
\hline Total no. of groups & No. of participants & Criteria \\
\hline Group-I & 57 & Individuals who were deliberately self-poisoned and admitted in the hospital. \\
Group-II & 57 & Healthy individuals. \\
\hline
\end{tabular}


7 - 10 days admission in the hospital. Once it was ensured that suitable subjects were selected for the study (Bilateral hearing sensitivity within normal limits and no middle ear abnormalities), the subjects were evaluated by Distortion Product Otoacoustic Emissions (DPOAE) testing. The results were evolved based on "pass" or "fail" criteria and the responses were considered as "pass" if 7 out of 9 frequencies were above $6 \mathrm{~dB}$ noise level.

\section{Results}

DPOAE findings revealed the Pass or Fail criteria in both the groups. The subjects who had deliberate ingestion of OP pesticides (Group-I) were found to have "Passed" criteria of 30\% (34 ears) and "Failed" criteria of 70\% (80 ears). The healthy subjects (Group-II) were found to have "Passed" criteria of 91\% (104 ears) and "Failed" criteria of $9 \%$ (10 ears). The failure result of DPOAE in the former group was bilaterally noticed which inferred the absence of DPOAE in both the ears. The results of 'Failure' criteria in DPOAE in Group-I (subjects who were selfpoisoned) compared to Group-II (Healthy subjects) and chi-square analysis had been done which was found to be statistically significant $(\mathrm{p}<0.01)$.

The following Bar-diagram (Figure 1) represents the comparison of DPOAE findings between the groups.

\section{Discussion}

The current study showed statistically significant failure of DPOAE in the subjects of group I which unfolds cochlear changes following pesticide ingestion. The two important pathologies behind OP toxicity are the generation of Reactive Oxygen Species (ROS) and the depletion of NADPH which is necessary for normal function [33]. A study [34] reported oxidative damage following OP poisoning. ROS are associated with drugs like cisplatin, amino glycosides [35,36] and noise-induced [37, 38] cochlear pathology. The authors suggested that the intoxication route of solvents in the inner ear would be

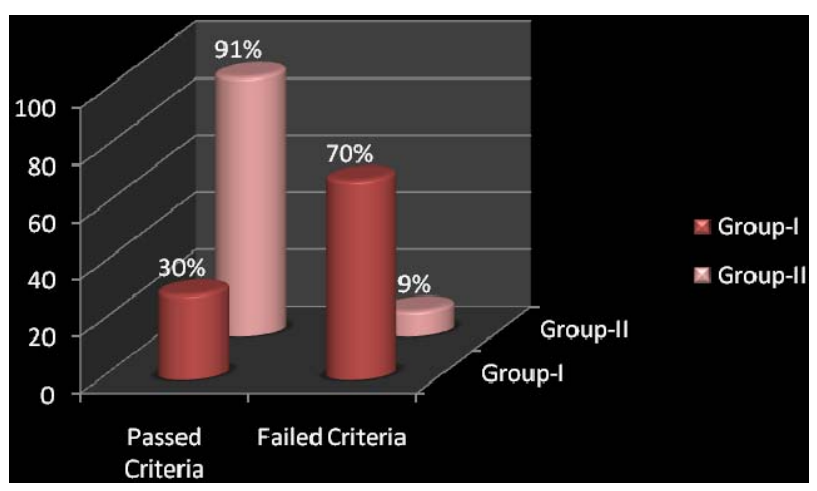

Figure 1. Comparison of DPOAE findings between the groups. via blood transport from the stria vascularis or the spiral prominence. Then, solvents would diffuse through the outer sulcus by the lipid-rich content of the membranes and reach the lipid-rich Hensen's cells. The latter cells are in close connection with the Deiter's cells, which are located under the OHCs, and thus the target is reached. However, the route of solvent-induced cochlear damage has not yet proved. Very few human studies have examined ototoxic effects with pesticide exposure but no studies have assessed the ototoxic effects following acute exposure to OP (even in deliberate oral ingestion conditions also). A study [39] was done on OP poisoned patients to know the ototoxic effects on ABR which concludes that the generation of ROS following the ingestion of OP, may not be sufficient to cause lesions in the auditory pathway but damage to the inner and outer hair cells cannot be excluded. Hence the current study explains the changes in cochlea which was represented by significant absence of DPOAE in the study group. These results suggest that exposure to pesticides and in particular, OP compounds, may contribute to hearing loss.

For clinical audiologists, there are two important issues to consider when assessing solvent-induced hearing loss [40]. The first is related to early detection. It is integral to the scope of practice for audiologists to diagnose a hearing loss in its initial stage, and thus reduce the chance of further, progressive damage with the adverse consequences on clients' quality of life. Another aspect of clinical practice for audiologists is the auditory rehabilitation and the enhancement of quality of life of persons with hearing impairment. The first stage of any auditory rehabilitation plan is a comprehensive evaluation of the auditory damage. Rehabilitation then focuses on those aspects of audition that are especially affected. For solvent-induced hearing loss, such characterization requires the use of tests sensitive to abnormalities of both peripheral and central auditory structures. Hence the present study states the importance of DPOAE in detecting the damage due to OP poisoning. The future research should be focussed on determining frequency specific responses of the DPOAE on the subjects, which will be of great use.

\section{Conclusion}

The current study concludes the significant changes in cochlear outer hair cells after self-poisoning with OP pesticide which was reflected by the significant failure in DPOAE in the subjects who were self-poisoned. So these agents may induce auditory damage not only in animals and but also in humans. Audiologists should be alert to the oto-toxic properties of OP compounds and conduct a comprehensive audiological assessment even in the conditions of deliberate ingestions. 


\section{Acknowledgements}

The authors are grateful to all the subjects who participated in the study. The authors would like to thank the entire unit of People's Education Society Institute of Medical Sciences and Research centre, Kuppam, Andhra Pradesh, India for permitting to collect the data. The authors would like to thank Indian Speech \& Hearing Association for giving us an opportunity to present this paper in the Annual National Conference 2012.

\section{REFERENCES}

[1] World Health Organisation (WHO), "Pesticides Are a Leading Suicide Method,” World Health Organisation, Geneva, 2006.

[2] National Health Services (NHS) Centre for Review and Dissemination, "Deliberate Self-Harm. Effective Healthcare Bulletin,” NHS Centre, Vol. 4, 1998, pp. 1-12.

[3] J. M. Bertolote, A. Fleischmann, M. Eddleston and D. Gunnell, "Deaths from Pesticide Poisoning: A Global Response,” The British Journal of Psychiatry, Vol. 189, 2006, pp. 201-203. doi:10.1192/bjp.bp.105.020834

[4] M. R. Phillips, G. Yang, Y. Zhang, et al., "Risk Factors for Suicide in China: A National Case-Control Psychological Autopsy Study,” Lancet, Vol. 360, No. 9347, 2002, pp. 1728-1736. doi:10.1016/S0140-6736(02)11681-3

[5] J. Jayarathnam, “Acute Pesticide Poisoning: A Major Global Health Problem,” World Health Statistics Quarterly, Vol. 43, 1990, pp. 139-144.

[6] M. Harell, J. J. Shea and J. R. Emmett, "Bilateral Sudden Deafness Following Combined Insecticide Poisoning," Laryngoscope, Vol. 88, 1978, pp. 1348-1351.

[7] C. S. Petty, "Organic Phosphate Insecticide Poisoning: Residual Effects in Two Cases," American Journal of Medicine, Vol. 24, No. 3, 1958, pp. 567-568.

[8] P. Campo, R. Lataye, B. B. Cossec and V. Placidi, "Toluene-Induced Hearing Loss: A Mid-Frequency Location of the Cochlear Lesions," Neurotoxicology and Teratology, Vol. 19, No. 2, 1997, pp. 129-40. doi:10.1016/S0892-0362(96)00214-0

[9] N. L. Cappaert, S. F. Klis, A. B. Baretta, H. Muijser and G. F. Smoorenburg, "Ethyl Benzene-Induced Ototoxicity in Rats: A Dose Dependent Mid-frequency Hearing Loss," Journal of the Association for Research in Otolaryngology, Vol. 1, 2000, pp. 292-299.

[10] T. C. Morata, A. C. Fiorini, F. M. Fischer, S. Colacioppo, K. M. Wallingford, et al., "Toluene-Induced Hearing Loss among Rotogravure Printing Workers,” Scandinavian Journal of Work, Environment Health, Vol. 23, No. 4, 1997, pp. 289-298. doi:10.5271/sjweh.222

[11] B. E. Moen, T. Riise and K. R. Kyvik, "P300 Brain Potential among Workers Exposed to Organic Solvents,” Norsk Epidemiologi, Vol. 9, 1999, pp. 27-31.

[12] D. T. Kemp, "Stimulated Acoustic Emissions from within the Human Auditory System," Journal of the Acoustical Society of America, Vol. 64, No. 5, 1978, pp. 1386-1391. doi:10.1121/1.382104

[13] D. T. Kemp, "Oto-Acoustic Emissions, Their Origin in Cochlear Function, and Use in Hearing and Balance," British Medical Bulletin, Vol. 63, No. 1, 2002, pp. 223241. doi:10.1093/bmb/63.1.223

[14] M. Riga, "The Effect of Treatment with Vincristine on Transient Evoked and Distortion Product Oto-Acoustic Emissions," International Journal of Pediatric Otorhinolaryngology, Vol. 70, No. 6, 2006, pp. 1003-1008. doi:10.1016/j.ijporl.2005.10.011

[15] R. Lataye, K. Maguin and P. Campo, "Increase in Cochlear Microphonic Potential after Toluene Administration,” Hearing Research, Vol. 230, No. 1-2, 2007, pp. 3442. doi:10.1016/j.heares.2007.04.002

[16] R. Lataye, P. Campo, G. Loquet and G. Morel, "Combined Effects of Noise and Styrene on Hearing: Comparison between Active and Sedentary Rats,” Noise Health, Vol. 7, No. 27, 2005, pp. 49-64. doi:10.4103/1463-1741.31633

[17] I. C. P. R. Russo and T. M. M. Santos, "A Prática da Audiologia clíNica,” Cortez, São Paulo, 1993.

[18] M. W. Yellin and R. D. Stillman, "Oto-Acoustic Emissions in Normal-Cycling Females," Journal of American Academy of Audiology, Vol. 10, 1999, pp. 400-408.

[19] R. Probst, F. P. Harris and R. Hauser, "Clinical Monitoring Using Oto-Acoustic Emissions,” British Journal of Audiology, Vol. 27, No. 2, 1993, pp. 85-90. doi:10.3109/03005369309077896

[20] P. Avan, J. D. Durrant and B. Buki, "Possible Effects of Cochlear Hydrops and Related Phenomenon on OAEs," Seminars in Hearing, Vol. 22, No. 4, 2001, pp. 405-414. doi:10.1055/s-2001-19113

[21] S. Frota, "Fundamentos em Fonoaudiologia: Audiologia. Rio de Janeiro: Guanabara Koogan,” 2003.

[22] M. A. Hotz, F. P. Harris and R. Probst, "Oto-Acoustic Emissions: An Approach for Monitoring Amino Glycoside-Induced Ototoxicity,” Laryngoscope, Vol. 104, 1994, pp. 1130-1134.

[23] K. C. M. Campbell and J. D. Durrant, "Audiological Monitoring for Ototoxicity," Otolaryngologic Clinics of North America, Vol. 26, 1993, pp. 903-914.

[24] K. M. Reavis, D. S. Phillips, S. A. Fausti, et al., "Factors Affecting Sensitivity of Distortion Product Oto-Acoustic Emissions to Ototoxic Hearing Loss,” Ear \& Hearing, Vol. 29, No. 6, 2008, pp. 875-893. doi:10.1097/AUD.0b013e318181ad99

[25] K. M. Reavis, et al., "Distortion-Product Otoacoustic Emission Test Performance for Ototoxicity Monitoring," Ear \& Hearing, Vol. 32, 2011, pp. 61-74.

[26] F. Ottaviani, N. Dozio, C. B. Neglia, S. Riccio and M. Scavini, "Absence of Oto-Acoustic Emissions in InsulinDependent Diabetic Patients: Is There Evidence for Diabetic Cochleopathy?” Journal of Diabetes and Its Complications, Vol. 16, No. 5, 2002, pp. 338-343. doi:10.1016/S1056-8727(01)00224-0

[27] Y. Zhang, X. Zhang, W. Zhu, X. Zheng and X. Deng, "Distortion Product of Oto-Acoustic Emissions as a Sensitive Indicator of Hearing Loss in Pilots,” Aviation, 
Space, and Environmental Medicine, Vol. 75, 2004, pp. 46-48.

[28] T. C. Morata and G. K. Lemasters, "Epidemiologic Considerations in the Evaluation of Occupational Hearing Loss," Occupational Medicine, Vol. 10, 1995, pp. 641656.

[29] B. Katbamna, D. N. Homnick and J. H. Marks, "Effects of Chronic Tobramycin Treatment on Distortion Product Oto-Acoustic Emissions,” Ear and Hearing, Vol. 20, No. 5, 1999, pp. 393-402. doi:10.1097/00003446-199910000-00002

[30] P. Stavroulaki, N. Apostolopoulos, J. Segas, M. Tsakanikos and G. Adam-opoulos, "Evoked Oto-Acoustic Emissions-An Approach for Monitoring Cisplatin Induced Ototoxicity in Children," International Journal of Pediatric Otorhinolaryngology, Vol. 59, No. 1, 2001, pp. 47-57. doi:10.1016/S0165-5876(01)00455-4

[31] B. D. Ress, K. S. Sridhar, T. J. Balkany, G. M. Waxman, B. B. Stagner and B. L. Lonsbury-Martin, "Effects of Cisplatinum Chemotherapy on Oto-Acoustic Emissions: The Development of an Objective Screening Protocol," Otolaryngology—Head \& Neck Surgery, Vol. 121, No. 6, 1999, pp. 693-701. doi:10.1053/hn.1999.v121.a101567

[32] P. Avan and P. Bonfils, "Distortion-Product Oto-Acoustic Emission Spectra and High-Resolution Audiometry in Noise-Induced Hearing Loss," Hearing Research, Vol. 209, No. 1-2, 2005, pp. 68-75. doi:10.1016/j.heares.2005.06.008

[33] J. S. Bus and J. E. Gibson, "Paraquat: Model for Oxidant-Initiated Toxicity," Environmental Health Perspectives, Vol. 55, 1984, pp. 37-46. doi:10.1289/ehp.845537

[34] M. Dandapani, A. Zachariah, M. R. Kavitha , L. Jeyasee- lan and A. Oommen, "Oxidative Damage in Intermediate Syndrome of Acute OrganoPhosphorous Poisoning," Indian Journal of Medical Research, Vol. 117, 2003, pp. 253-259.

[35] W. J. Clerici and L. Yang, "Direct Effects of Intraperilymphatic Reactive Oxygen Species Generation on Cochlear Function,” Hearing Research, Vol. 101, No. 1-2, 1996, pp. 14-22. doi:10.1016/S0378-5955(96)00126-8

[36] E. M. Priuska and J. Schacht, "Formation of Free Radicals by Gentamicin and Iron and Evidence for an Iron/ Gentamicin Complex,” Biochemical Pharmacology, Vol. 50, No. 11, 1995, pp. 1749-1752. doi:10.1016/0006-2952(95)02160-4

[37] K. C. Harris, E. Bielefeld, B. H. Hu and D. Henderson, "Increased Resistance to Free Radical Damage Induced by Low-level Sound Conditioning," Hearing Research, Vol. 213, 2006, pp. 118-129. doi:10.1016/j.heares.2005.11.012

[38] K. K. Ohlemiller, J. S. Wright and L. L. Dugan, "Early Elevation of Cochlear Reactive Oxygen Species Following Noise Exposure,” Audiology \& Neurootology, Vol. 4, No. 5, 1999, pp. 229-236. doi:10.1159/000013846

[39] S. S. Jayasinghe and K. D. Pathirana, "Effects of Deliberate Ingestion of Organo Phosphate or Paraquat on BrainStem Evoked Potentials,” Journal of Medical Toxicology, Vol. 7, No. 4, 2011, pp. 277-280. doi:10.1007/s13181-011-0173-3

[40] A. Fuente and B. McPherson, "Organic Solvents and Hearing Loss: The Challenge for Audiology,” International Journal of Audiology, Vol. 45, No. 7, 2006, pp. 367-381. doi:10.1080/14992020600753205 